\title{
Positional aphasia as a manifestation of syndrome of the trephined: illustrative case
}

\author{
Erica C. Johnson, BS, Patrick F. O'Brien, MD, and William C. Broaddus, MD, PhD \\ Department of Neurosurgery, Virginia Commonwealth University Health System, Richmond, Virginia
}

BACKGROUND The authors report a case of a 66-year-old male who presented acutely with a subdural hematoma who was managed operatively with craniotomy. His course was complicated by a postoperative epidural hematoma, which, on the basis of intraoperative findings at the second surgery, was managed with evacuation of the hematoma and removal of the bone flap.

OBSERVATIONS The patient's subsequent recovery was remarkable for a reproducible positional aphasia in the early postoperative period with an ultimate diagnosis of syndrome of the trephined. The patient's cerebral edema permitted early autologous cranioplasty, which resulted in resolution of the patient's symptoms.

LESSONS The authors believe this case to be the first described of isolated positional aphasia as a manifestation of syndrome of the trephined. Recognition and treatment of the syndrome resulted in a positive patient outcome.

https://thejns.org/doi/abs/10.3171/CASE21629

KEYWORDS syndrome of the trephined; decompressive hemicraniectomy; complications; positional aphasia

The "syndrome of the trephined" or "sinking skin flap syndrome" is a rare complication of a craniectomy characterized by postoperative neurological deterioration caused by cortical dysfunction of the area below the craniotomy that improves after cranioplasty. ${ }^{1}$ Ashayeri et al. ${ }^{2}$ published a review in 2016 based on 54 cases that found this syndrome can occur in adults at any age, with an average reported age of 49 years and a male predominance $(60 \%)$. This phenomenon was most often found after craniectomy for treatment of neurotrauma, and the time to onset of symptoms after surgery was $5.1 \pm 10.8$ months after surgery. $^{2}$ This review also described presenting symptoms in order of frequency. The most common presenting symptoms included motor weakness, cognitive deficits, and language deficit.

A review published in 2015 in the British Journal of Neurosurgery presented similar findings in terms of presenting symptoms. One article reported motor symptoms in 17 of 22 cases. Cognitive symptoms were also discussed and included bradypsychia, slowing of speech fluency, impaired naming and word finding, and several others. ${ }^{3}$ This review also discussed time between craniectomy and onset of symptoms. Across the whole review of 83 patients, the reported mean time before neurological decline was 50 weeks, with a significant range, and 4 cases described appearance of symptoms within several days after craniectomy. ${ }^{3}$

The pathophysiology of this syndrome is incompletely understood, but several theories have been proposed. The first is that the cranial defect allows atmospheric pressure to be transmitted to the cerebral vasculature, causing decreased cerebral blood flow to the area beneath the defect that precipitates the symptoms. ${ }^{4}$ Another theory involves cerebrospinal fluid (CSF) dynamics and suggests that the cerebral injury causes alterations in CSF flow, leading to transgression of CSF and edema in the brain parenchyma below the skull defect. It is thought that normal flow dynamics are restored after cranioplasty. ${ }^{2}$ We present a case of positional aphasia as the solitary manifestation of the syndrome of the trephined that became apparent on postoperative day 3 after decompressive craniectomy for an epidural hematoma.

\section{Illustrative Case}

The patient was a 66-year-old man with a previous medical history of a myocardial infarction and hypertension being treated with aspirin

ABBREVIATIONS CSF = cerebrospinal fluid; $C T$ = computed tomography; EEG = electroencephalography. INCLUDE WHEN CITING Published February 14, 2022; DOI: 10.3171/CASE21629.

SUBMITTED November 3, 2021. ACCEPTED December 6, 2021.

(c) 2022 The authors, CC BY-NC-ND 4.0 (http://creativecommons.org/licenses/by-nc-nd/4.0/). 

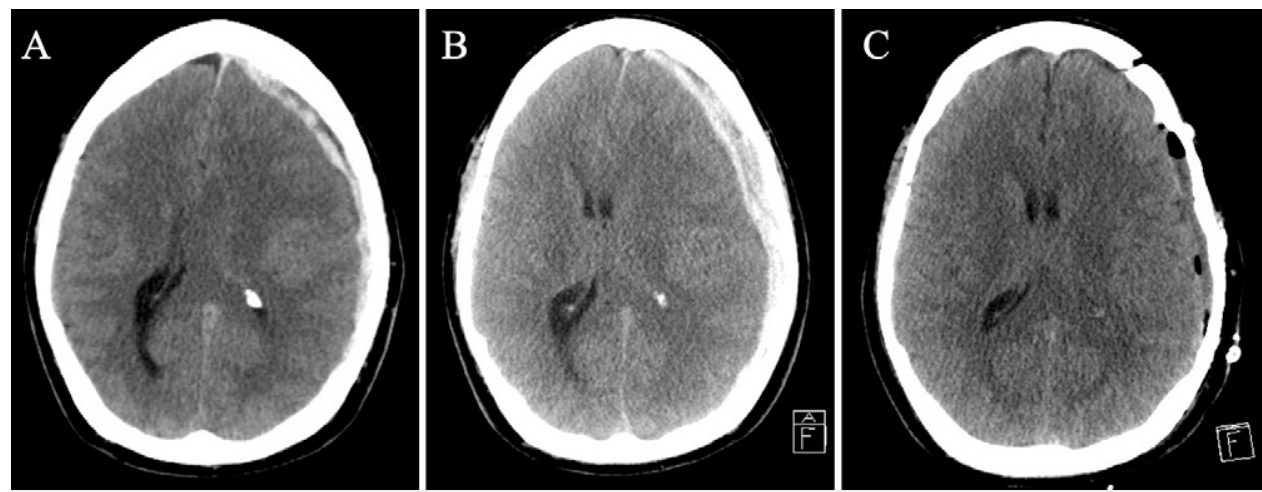

FIG. 1. Initial (A) and repeat (B) CT scans of the head showing the patient's epidural hematoma. C: Postoperative CT scan after hematoma evacuation.

who presented to a local hospital with 3 days of progressively worsening headaches and an unknown history of trauma. Non-contrastenhanced computed tomography (CT) of the head was performed at the initial hospital, which showed a left-sided subdural hematoma with $6 \mathrm{~mm}$ of midline shift (Fig. 1A). He was then transferred to Virginia Commonwealth University Health System for definitive management. A repeat head CT scan was obtained upon arrival (Fig. 1B), which showed increased left-to-right midline shift, and the patient was taken immediately to the operating room for craniotomy for evacuation of the hematoma. Per protocol, a postoperative head CT scan was obtained, which showed resolution of the hematoma (Fig. 1C).

His postoperative course was complicated 2 days later, when he was noted to have difficulty speaking and decreased movement of the right upper extremity. A CT scan of the head was obtained, which showed a new left-sided epidural hematoma (Fig. 2, left). He was taken back to the operating room with the intent to perform a redo craniotomy and evacuation of the epidural hematoma. The operation was converted to a hemicraniectomy due to the degree of cerebral edema encountered during the surgery. The patient had postoperative improvement in his speech and motor function. Per our postoperative routine, the patient was initiated on prophylactic levetiracetam for a planned 7-day course. A postoperative head CT scan was again obtained, showing resolution of the epidural hematoma and the craniectomy defect (Fig. 2, right).
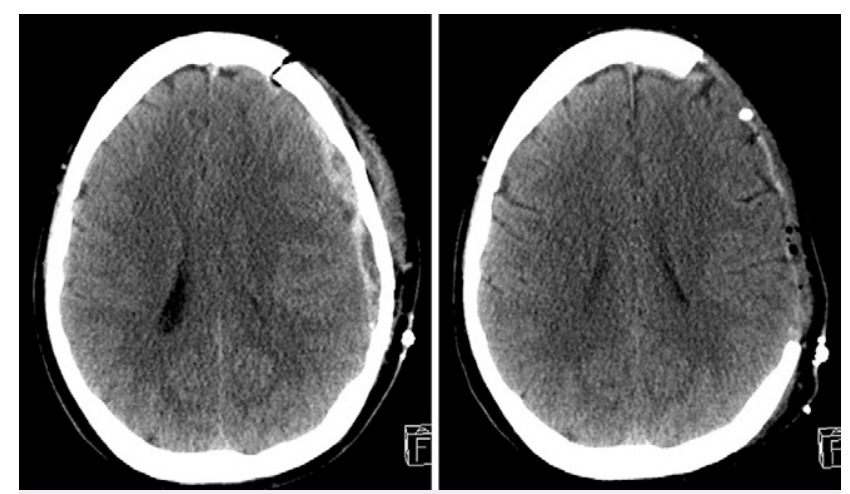

FIG. 2. Left: Repeat head CT scan obtained with recurrent epidural hematoma. Right: Postoperative CT scan after hemicraniectomy and hematoma evacuation.
The patient remained at this neurological baseline until 2 days after his second operation (postoperative day 5 from the index surgery), when he was found to be acutely expressively aphasic without evidence of receptive dysfunction or motor deficit or drift. Workup for the decline in the patient's examination findings included another CT scan of the head, which showed expected postoperative changes without acute findings. He was reloaded with levetiracetam with escalation of his maintenance dosing, given concern for possible seizures. Immediately, continuous video electroencephalography (EEG) was performed, which showed generalized slowing with focal delta slowing on the left consistent with breach artifact but no seizure activity; there was no specific comment about postural EEG changes on this study. Further work-up for ischemia was undertaken, including CT angiography and CT perfusion (Fig. 3) and magnetic resonance imaging with and without contrast (Fig. 4); the findings of these studies were also found to be negative. Of note, the patient's scalp flap was noted on serial physical examinations to be sunken below the margins of the hemicraniectomy after being upright for a period of 10-15 minutes. After the patient had been recumbent for 10-15 minutes, the scalp flap was fuller and not sunken. The EEG remained in place for 2 days with a working diagnosis of seizure before initiation of EEG recording.

After this work-up, the patient continued to exhibit an intermittent expressive aphasia despite the escalation of antiepileptic therapy and the absence of seizures on the EEG. It was noted that symptoms consistently followed being upright during the daytime and correlated with a sunken appearance of the scalp flap. His aphasia was noted to be markedly improved after he was supine for a time, such as after awakening in the morning or after being placed supine when the aphasia developed. The improvement also correlated with a less sunken appearance of the scalp flap in the recumbent position. After repeated observations with reproducible clinical findings were demonstrated, a putative diagnosis of syndrome of the trephined was further explored. The patient underwent 2 more days of continuous video EEG monitoring with specific attention to postural changes in EEG, with no such changes identified. With alternative diagnoses ruled out, the patient was diagnosed with syndrome of the trephined. The patient's cerebral edema was clinically improved on the basis of bedside examination demonstrating a persistently sunken craniectomy site when upright, so he underwent autologous cranioplasty on postoperative day 12 from the index operation. His course after cranioplasty was uncomplicated. The 

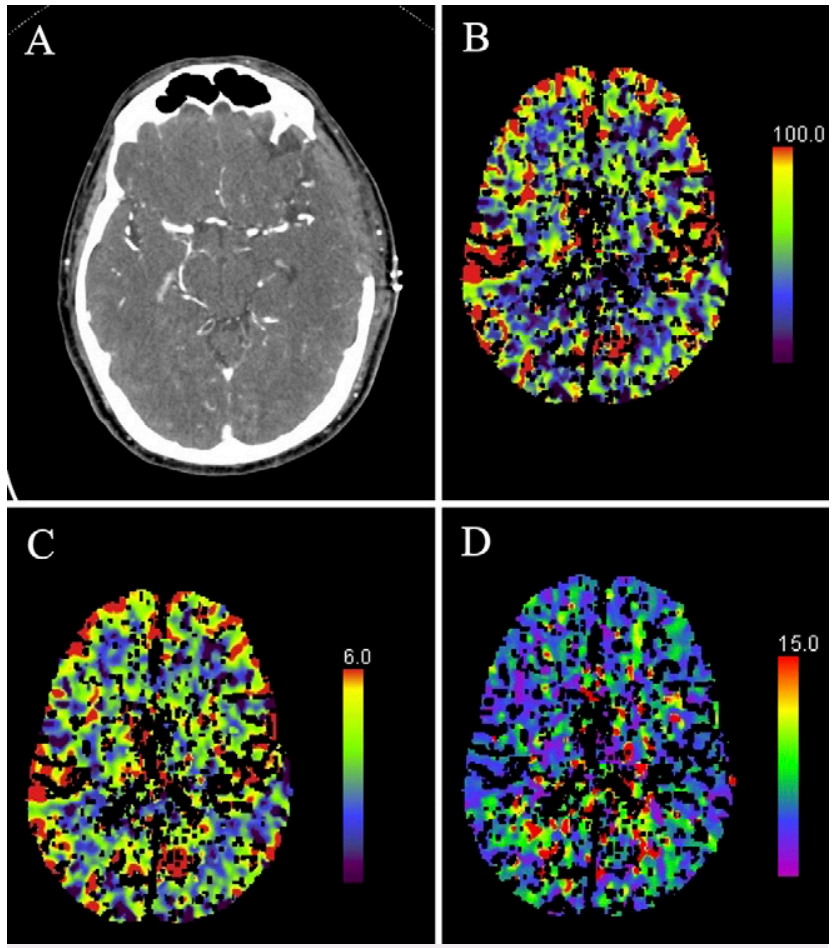

FIG. 3. CT angiography (centered on circle of Willis) (A), CT perfusion cerebral blood flow (B), CT perfusion cerebral blood volume (C), and CT perfusion time to drain (D) shows no territorial perfusion deficits.

finding of postoperative imaging was stable (Fig. 5). The patient demonstrated resolution of his positional aphasia, and he was discharged home the following day. In his follow-up examination 3 months after discharge, the patient continued to do well neurologically with speech having returned to baseline and no wound or flap complications.

\section{Discussion}

\section{Observations}

The syndrome of the trephined is a rare phenomenon whose etiology and manifestations have yet to be fully described in the literature. We present a case of a patient who became symptomatic several days after decompressive craniectomy for a subdural hematoma. His symptoms were primarily related to expressive speech and found to be positional in nature, worse while sitting with improvement while the patient was in the supine position. Recognition of the positional nature of his symptoms prompted expeditious reimplantation of the autologous bone flap, with resolution of his symptoms.

The underlying etiology of the patient's initial subdural hematoma remains uncertain. The patient was receiving antiplatelet medications before arrival with no documented antecedent trauma. It is possible he sustained an occult minor trauma that was not recollected or witnessed. The imaging studies obtained ruled out hemorrhage from underlying pathological vascular lesion. CSF hypotension of an alternative cause, such as a spontaneous CSF leak, could be entertained as a possible etiology, although generally this complication is reported in association with lumbar drainage or shunting, and subdural hematoma secondary to CSF overdrainage is rare. ${ }^{5}$
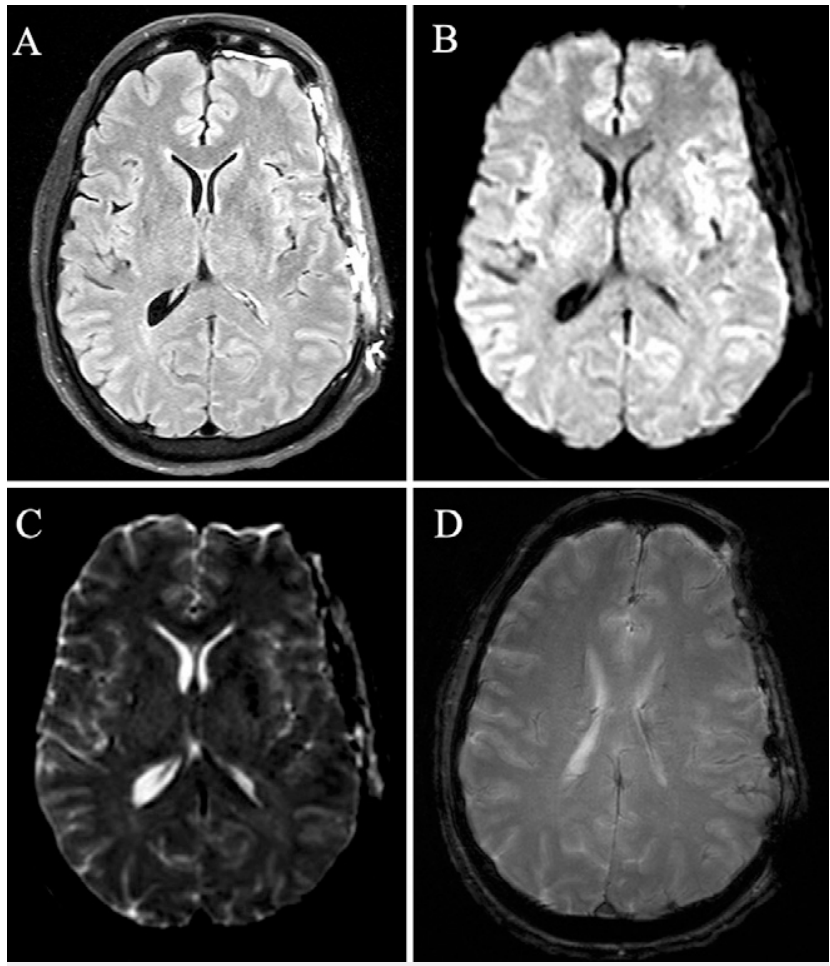

FIG. 4. Magnetic resonance imaging of the brain. T2-weighted fluidattenuated inversion recovery image $(\mathbf{A})$, axial diffusion-weighted image (B), axial apparent diffusion coefficient image (C), and axial gradient recalled echo image (D) show no explanatory stroke or mass lesion to explain the patient's aphasia.

\section{Lessons}

Although positional aphasia has not previously been reported as a consequence of decompressive craniectomy overlying the dominant hemisphere, this case illustrates the potential benefits of early reimplantation of the decompressive bone flap after adequate

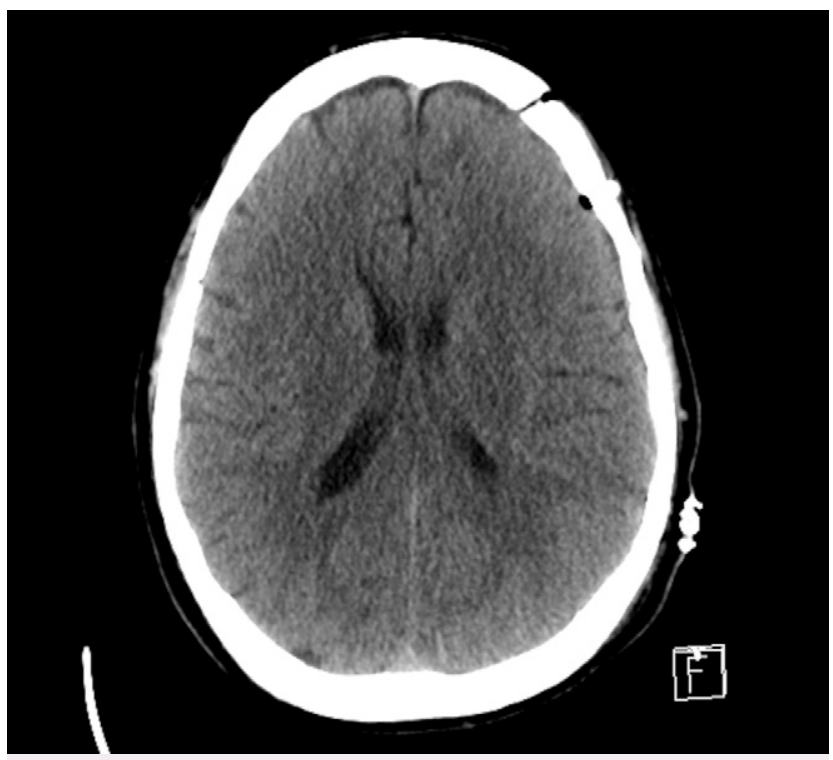

FIG. 5. Post-cranioplasty head CT scan. 
resolution of the underlying brain edema, particularly when there may be adverse neurological effects due to the cranial defect.

\section{References}

1. Krishnan P, Chowdhury SR. Posture-dependent aphasia: focal cortical dysfunction in the sinking scalp flap syndrome. J Neurosci Rural Pract. 2015;6(2):225-227.

2. Ashayeri K, Jackson EM, Huang J, Brem H, Gordon CR. Syndrome of the trephined: a systematic review. Neurosurgery. 2016;79(4):525-534.

3. Annan M, De Toffol B, Hommet C, Mondon K. Sinking skin flap syndrome (or syndrome of the trephined): a review. $\mathrm{Br} J$ Neurosurg. 2015;29(3):314-318.

4. Joseph V, Reilly P. Syndrome of the trephined: case report. J Neurosurg. 2009;111(4):650-652.

5. Mokri B. Cerebrospinal fluid volume depletion and its emerging clinical/imaging syndromes. Neurosurg Focus. 2000;9(1):e6.

\section{Disclosures}

The authors report no conflict of interest concerning the materials or methods used in this study or the findings specified in this paper.

\section{Author Contributions}

Conception and design: all authors. Acquisition of data: Johnson, O'Brien. Analysis and interpretation of data: all authors. Drafting the article: Johnson. Critically revising the article: all authors. Reviewed submitted version of manuscript: all authors. Approved the final version of the manuscript on behalf of all authors: Broaddus. Study supervision: Broaddus.

\section{Correspondence}

William C. Broaddus: Virginia Commonwealth University Health System, Richmond, VA. william.broaddus@vcuhealth.org. 